determine the frequency of detection of $\mathrm{M}$. genitalium and to investigate causal relationship between $\mathrm{M}$. genitalium and cervicitis.

Methods Two hundred women who attended gynecologic clinics were recruited. Mucopurulent cervicitis was defined as presence of either visible yellow mucopus or presence of > 30PMNL/1000 X microscopic field on gramme stain smear of cervical mucus. First void urine (FVU), three endocervical swabs (ECS) were collected from the study group and control group. All the samples were tested by PCR amplification for presence of $\mathrm{M}$. genitalium by targeting $\mathrm{MgPa}$ gene as described previously. Samples were also subjected to culture for other genital Mycoplasmas and PCR amplification for Chlamydia trachomatis and Neisseria gonorrhoeae.

Results Mycoplasma genitalium was found in 6\% from ECS and $5 \%$ from Urine of women with Cervicitis and overall M. genitalium was detected in $11 \%$ of women and $1 \%$ from Controls $(\mathrm{P}<0.05)$. Disordered proliferative endometrium was observed in 4 of the $\mathrm{M}$. genitalium positive cases. All the patients who were positive for Chlamydia trachomatis (5\%) and Mycoplasma hominis $(7 \%)$ and Ureaplasma urealyticum(4\%) were excluded from the study. Prior miscarriage, menstrual cycle, whitish vaginal discharge and pelvic discomfort were positively associated with $\mathrm{M}$. genitalium.

Conclusions The study suggests association of $M$. genitalium infection and Cervicitis and this microorganism should be routinely screened in patients of cervicitis.

\section{P3.295 RISK BEHAVIOR AND SEXUALLY TRANSMITTED INFECTIONS AMONG PEOPLE LIVING WITH HIV/AIDS IN BELIZE, 2012}

doi:10.1136/sextrans-2013-051184.0750

'S Morales-Miranda, ${ }^{2} \mathrm{M}$ Manzanero, 'I Loya, 'R Mendizabal, 'B Alvarez. 'Universidad del Valle de Guatemala, Guatemala, Guatemala, ${ }^{2}$ Ministry of Public Health, Belize, Belize

Background STIs among people living with HIV/AIDS (PLHIV) have a direct implication on spread of HIV and the effectiveness of prevention programmes. For the first time, risk behaviour and STI prevalence was determined for PLHIV in Belize, as part of the 2012 Central American Behavioral Surveillance Survey of HIV/STI.

Methods Participants were selected based on convenience sampling of active PLWHA in the Belize Health Information System. Data collection consisted of a behavioural survey questionnaire administred through audio computer-assisted self-interview (ACASI). A blood sample was drawn for syphilis and herpes simplex virus type 2 (HSV-2) testing. Additionally, genital samples were tested for Treponema pallidum (TP), Chlamydia trachomatis (CT), Neisseria gonorrhoeae (NG), Trichomonas vaginalis (TV) and Mycoplasma genitalium (MG). Participation was voluntary and anonymous, signed consent was required. Crude proportions for categorical variables and medians and interquartile range (IOR) for numerical variables were calculated using STATA 9.0.

Results A total of 252 PLHIV were enrolled (57\% female), from three districts of Belize (concentrating 80\% of the PLHIV in the country). Median age was 45 years old (IOR 28-42). Long-standing diagnosed HIV infection was detected, median 5 years (IOR 2.3-8). Low monthly income (< US\$360) was found in $85 \%$ females and $50 \%$ males, $37.0 \%$ reported no current stable partners or occasional partners in the last year. Low percentage of consistent condom use with stable partners ( $40 \%$ female, $47.7 \%$ male) was reported. A high prevalence for HSV-2 (82.86\%), followed by TV (40.3\% female and $2.25 \%$ male) and MG (13.2\% female and $17.98 \%$ male) and a low prevalence for syphilis (1.6\%) and NG was observed (1.12\%).

Conclusions Results from the first behavioural and biological survey among PLHIV demonstrate a need for implementation of a specific Sexual Health Program for this population. This programme would include education promoting a healthy sexual lifestyle, and regular distribution of condoms.
P3.296 PREVALENCE OF CHLAMYDIAL INFECTIONS WITHIN EIGHT SOUTH AFRICAN PROVINCES (2006-2011)

doi:10.1136/sextrans-2013-051184.0751

F Radebe, V Maseko, I Basson, G de Gita, S Takuva, D A Lewis. Centre for HIV \& STIS, NICD/NHLS, Johannesburg, South Africa

Background The microbiological surveillance was undertaken in eight provinces of South Africa during 2006-2011 to determine the aetiology of the male urethritis syndrome (MUS), vaginal discharge syndrome (VDS) and genital ulcer syndrome (GUS) and the prevalence of HIV, HSV-2 and syphilis.

Methods 1361 MUS, 1691 VDS and 465 GUS cases were consecutively recruited in eight South African provinces (2006-2011). Laboratory-based diagnostic methods included nucleic acid amplification to detect Chlamydia trachomatis, Neisseria gonorrhoeae, Mycoplasma genitalium, Trichomonas vaginalis, Herpes Simplex Virus 2, Haemophilus ducreyi, Treponema pallidum and Chlamydia trachomatis serovars L1-3.

Results Overall, 202 (14.9\%) MUS and 240 (14.2\%) VDS cases were positive for $C$. trachomatis while $6(1.3 \%)$ GUS cases were positive for $C$. trachomatis serovars L1-3. The highest prevalence of $C$. trachomatis was $21.1 \%$ in Gauteng among men and $19.4 \%$ in women. The prevalence in other provinces was: Mpumalanga (men 18.4\%; women 17.4\%), Limpopo (men 14.0\%; women 16.7\%), Eastern Cape (men 16.4\%; women 13.5\%), Western Cape (men 13.5\%; women $14.9 \%$ ), Northwest (men 10.3\%; women 11.1\%), Free State (men 8.0\%; women 9.8\%) and Northern Cape (men 8.1\%; women 9.6\%). C. trachomatis serovars LI-3 prevalence was 3.2\% in the Free State, $2.8 \%$ in Mpumalanga and $0.7 \%$ in Gauteng. No C. trachomatis serovars L1-3 were detected in other five provinces.

Conclusions The prevalence of $C$. trachomatis infection was high in this population and remains an important cause of genital infection in South Africa particularly in men. This may fuel the HIV epidemic which was high in most of the provinces in this study.

\section{P3.297 SURVEILLANCE OF SYPHILIS IN THE STATE OF SAO PAULO, BRAZIL}

doi:10.1136/sextrans-2013-051184.0752

C G Luppi, W K Alencar, M A Silva, S G E Chabu, S Romera. Centro de Referência de DST/AIDS-SES-SP, São Paulo, Brazil

Background The programme of STD/AIDS in the state of São Paulo has been implementing several actions toward to control and prevent sexually transmitted infections (STI): - congenital syphilis elimination plan, - access to diagnosis and treatment of acquired syphilis; - availability of syphilis rapid test in STD clinics. The report of syphilis confirmed cases was included in the Brazilian surveillance notification system since 2010, but the surveillance of STIs in the state of São Paulo began in 1998. The objective of this study is to describe the occurrence of syphilis cases reported in the state of São Paulo.

Methods It was performed a series of all cases reported in the state of São Paulo from 1998 to June 2012.

Results From 2007 42,965 cases were reported, 59\% were male, $37 \%$ young adults, $45 \%$ had schooling up to 8 years; $49 \%$ selfreported their race/ethnicity as white. From 1998 to 20034124 cases were reported, 1447 in 2006, and 10,022 cases in 2011. The number of cases increased approximately 7 times from 2006 to 2011. It was found an increase of 132\% (586/2007-1363/2011) in the number of services which has sent the syphilis notification.

Conclusion There was a significant increase of syphilis cases reported during this period analysed in the state of Sao Paulo. This result could be explained for: - inclusion of this condition on the national list of notification, - adherence of surveillance teams to the syphilis notification, - increase of syphilis rapid test in STD services. 
The increase in the number of health facilities as well as the number of cases could reflect in part the positive impact of actions implemented by the state programme.

\section{P3.298 TRENDS AND ASSOCIATIONS OF TRICHOMONAS VAGINALIS INFECTION IN MEN AND WOMEN WITH GENITAL DISCHARGE SYNDROMES IN JOHANNESBURG, SOUTH AFRICA}

doi:10.1136/sextrans-2013-051184.0753

'D V Maseko, ${ }^{2} \mathrm{~K}$ Marsh, ${ }^{1} \mathrm{~F}$ Radebe, ${ }^{2} \mathrm{G}$ Hughes, ${ }^{1,3,4} \mathrm{D}$ A Lewis. ${ }^{1}$ Centre for HIV and STIs, National Institute for Communicable Diseases, National Health Laboratory Service, Johannesburg, South Africa; ${ }^{2}$ IIV and STI Department, Health Protection Services Colindale, London, UK; ${ }^{3}$ Department of Internal Medicine, Faculty of Health Sciences, University of the Witwatersrand, Johannesburg, South Africa; ${ }^{4}$ Division of Medical Microbiology, University of Cape Town, Cape Town, South Africa

Objectives To better understand the epidemiology of Trichomonas vaginalis infection, we investigated the association between $T$. vaginalis and demographic, clinical, microbiological and behavioural characteristics of patients presenting with genital discharges to a primary healthcare clinic in Johannesburg, South Africa.

Methods During six annual surveys (2007-2012), 1,218 male urethral discharge syndrome (MUDS) and 1,232 vaginal discharge syndrome (VDS) cases were consecutively recruited. Diagnostic methods included nucleic acid amplification (Neisseria gonorrhoeae, Chlamydia trachomatis, T. vaginalis, Mycoplasma genitalium), microscopy (bacterial vaginosis, Candida) and serology (Treponema pallidum, HSV-2, HIV). Chi-squared tests and logistic regression analyses were used to identify predictors of $T$. vaginalis infection.

Results T. vaginalis prevalence decreased from 2007 to 2012 (men, $13.4 \%$ to $4.8 \%$, p < 0.001 ; women, $33.8 \%$ to $23.1 \%$, p < 0.001 ). Overall, $74(6.1 \%)$ men and 291 (23.6\%) women were T. vaginalis positive, with the highest prevalence in those aged $\geq 40$ years (men, $13.6 \%$; women, $30.9 \%$ ). T. vaginalis infection occurred more often in pregnant women (adjusted odds ratio, aOR, 2.67; 95\% confidence intervals, CI, 1.29-5.54) and women with serological evidence of $T$. pallidum (aOR, 1.63; 95\% CI 1.08-2.45) or HSV-2 infections (aOR $1.75 ; 95 \%$ CI 1.16-2.64). T vaginalis infection occurred less often in men with co-existent gonorrhoea (aOR $0.35,95 \% \mathrm{CI}, 0.21-0.57$ ) and in women with either BV (aOR 0.60, 95\% CI 0.44-0.82) or Candida morphotypes (OR 0.61, 95\% CI 0.43-0.86).

Conclusions Although the prevalence of $T$. vaginalis infection decreased over time, it remains an important cause of genital discharge in South Africa, particularly in older patients and pregnant women.

\section{P3.299 TETRACYCLINE RESISTANCE IN UREAPLASMA SPECIES ISOLATED FROM WOMEN PRESENTING FOR TERMINATION OF PREGNANCY IN PRETORIA, SOUTH AFRICA}

doi:10.1136/sextrans-2013-051184.0754

M Le Roux, B E De Villiers, M R M Ditsele, S T Monokoane, L M Ngobeni. University of Limpopo (Medunsa Campus), Ga-Rankuwa, South Africa

Background The risks of untreated sexually transmitted infections in association with termination of pregnancy are known to increase the risk of post-termination complications. Local studies have shown a high genital tract carriage of mycoplasmas. Tetracyclines are widely used as first line agents against when ureaplasmal infection is detected However, there is limited data on the susceptibility profiles and mechanisms of resistance amongst Ureaplasma strains circulating in the Pretoria community. This study was undertaken to determine antimicrobial resistance to tetracycline among Ureaplasma species isolated from women presenting for termination of pregnancy at a tertiary referral hospital in South Africa.
Methods Two vaginal swabs were collected from each of 100 women after written informed consent was obtained. The first swab was used for PCR detection of genital ureaplasmas and tetracycline- resistance genes. The second swab was used for culture (Mycoplasma Duo kit) and phenotypic antimicrobial susceptibility testing (SIR Mycoplasma kit).

Results Ureaplasma species were isolated from 46 women (46\%). Forty-two women were infected with $U$. urealyticum, 1 with $U$. parvum and 3 were dually infected. Susceptibility profiles were obtained for 41 isolates. Fifteen $(36.6 \%)$ demonstrated resistance to tetracycline. Fourteen (34.1\%) were also resistant to doxycycline with 1 isolate showing intermediate resistance. All 41 strains contained the tet $M$ gene, 39 contained the $1.7 \mathrm{~kb}$ fragment of the tetracycline resistance gene and 36 strains contained the int-Tn gene.

Conclusion Genital ureaplasmas were isolated from nearly half the study population of women presenting for TOP. The predominant species identified was $U$ urealyticum. Tetracycline and doxycycline resistance was detected nearly a third of the isolates and this has implications for management of patients to prevent post-partum complications. The study will be expanded and communicated to the National Department of Health as this will impact future strategies of intervention in this country.

\section{P3.300 ASSESMENT OF SEXUAL BEHAVIOUR OF ALBANIAN HIGH SCHOOL STUDENTS}

doi:10.1136/sextrans-2013-051184.0755

'A Harxhi, ${ }^{2} \mathrm{~L}$ Merkuri, ${ }^{3} \mathrm{E}$ Agolli, ${ }^{4} \mathrm{~A}$ Agolli, ${ }^{5} \mathrm{~A}$ Subashi. ${ }^{1}$ University Hospital Center of Tirana, Tirana, Albania; ${ }^{2}$ Institute of Public Health, Tirana, Albania; ${ }^{3}$ UNFPA office, Tirana, Albania; ${ }^{4}$ Faculty of Social Science, Tirana, Albania; ${ }^{5}$ University Vitrina, Tirana, Albania

Background The aim of this study is to assess the sexual behaviour of high school youngsters in Albania in order to design better behaviour change interventions targeting this group of young people.

Methods This is a quantitative behavioural survey targeting young pupil attending high schools carried out in all regions in Albania. A representative sample of 2172 pupils of age group 15-19 years old was included in the study. A stratified random sampling technique was used to obtain this sample. A self administered structured questionnaire was used to collect data. Data analysis was made using SPSS version 12.

Results Almost $30 \%$ of participants of the study reported having had sexual intercourse, with a median age at first intercourse of 15 years. Only $23 \%$ of those experiencing sexual intercourse have ever used a condom. The reason for using it is to prevent an unwanted pregnancy and not to prevent sexually transmitted infections. $90 \%$ of the young people identified pharmacy stores as the only place to find condoms, while the family planning centres are not considered at all. Majority of young people did not discuss issues regarding condom use with their sexual partners.

Conclusion Although sexual education is part of the school curricula since few years, further strategies and interventions are needed to address issues regarding delaying first sexual intercourse and increasing condom use among high school young people in Albania.

\section{P3.301 MALE CLIENTS OF SEX WORKERS IN THE UNITED STATES: CORRELATES WITH STI/HIV RISK BEHAVIORS AND URBANIZATION LEVEL}

doi:10.1136/sextrans-2013-051184.0756

M A Villarroel. Johns Hopkins Bloomberg School of Public Health, Baltimore, MD, United States

Background Men who have purchased sex from sex workers ("clients") have been associated with an increased risk of acquiring STIs including HIV and in influencing the spread of infection in the 